\title{
Comparison of identification and antimicrobial resistance pattern of Staphylococcus aureus isolated from Amassoma, Bayelsa state, Nigeria.
}

\author{
Funmilola Abidemi Ayeni ${ }^{1}$, Tombari Gbarabon ${ }^{2}$, Camilla Andersen ${ }^{3}$, Niels Nørskov-Lauritsen ${ }^{3}$
}

1. University of Ibadan, Faculty of Pharmacy, Department of Pharmaceutical Microbiology. Ibadan, Nigeria

2. Niger Delta University, Faculty of Pharmacy, Department of Pharmaceutical Microbiology, Amassoma, Nigeria 3. Aarhus University Hospital, Department of Clinical Microbiology, Aarhus, Denmark

\begin{abstract}
Background: Staphylococcus aureus is often responsible for fatal infections and recent upsurge of resistant strains has resulted in therapeutic failure. The identification of this microorganism is a major challenge to medical microbiologists in developing countries.

Methods: One hundred and eighty five isolates which had been previously isolated from the nares of 185 healthy college students' volunteers in Amassoma, Bayelsa State, South Nigeria were identified by MALDI TOF mass spectrometry, and PCR amplification of the spa gene. The identified isolates were compared with presumptive identities obtained by growth on MSA, tube coagulation and slide agglutination tests. Antimicrobial susceptibility testing of S. aureus isolates was performed by Kirby Bauer technique while MRSA was screened for by growth on chromIDTM MRSA plate and confirmed by PCR-amplification of $\mathrm{mecA} / \mathrm{mecC}$ genes.

Results: From the 185 staphylococci that grew with yellow colonies on MSA, 24 were positive in the slide coagulase test, while 17 were positive in the tube coagulase test; MALDI TOF mass spectrometry and PCR amplification of the spa gene showed excellent concordance with the tube test, as all tube coagulase-positive strains were identified as $\mathrm{S}$. aureus, while tube coagulase-test negative isolates in all cases were designated as other staphylococcal species by MALDI-TOF mass spectrometry and were spa PCR test negative. All S. aureus isolates were susceptible to clindamycin, vancomycin, fusidic acid, rifampicin and linezolid, while observed resistance to penicillin and trimethoprim were high. Only one MRSA strain was detected

Conclusion: The study confirms that the tube coagulase test is an accurate diagnostic method for identification of S. aureus, while growths on MSA and slide agglutination tests are inaccurate. We found a low prevalence of MRSA and a high rate of trimethroprim-resistance in the studied population.

Keywords: Antibiotics, coagulase, identification, S. aureus.

DOI: http://dx.doi.org/10.4314/ahs.v15i4.30

Cite as: Ayeni FA, Gbarabon T, Andersen C, Norskov-Lauritsen N. Comparison of identification and antimicrobial resistance pattern of Staphylococcus aureus isolated from Amassoma, Bayelsa state, Nigeria. Afri Health Sci. 2015;15(4):1282-8. http:/ /dx.doi.org/10.4314/ abs.v15i4.30
\end{abstract}

\section{Introduction}

Staphylococcus aureus is an important pathogen causing a wide range of infections e.g abscesses, furuncles, osteomyelitis, infective endocarditis and bacteremia. It is a major cause of severe nosocomial and community-acquired infections ${ }^{1}$. Its carriage in the anterior nares, considered to be the ecologic niche, plays a key role in the epidemiology of this pathogen ${ }^{2}$.

\section{Corresponding author:}

Funmilola Abidemi Ayeni, University of Ibadan, Department of Pharmaceutical Microbiology Email: funmiyeni@yahoo.co.uk
S. aureus can acquire resistance to many anti-microbial agents. Of particular concern is the synthesis of penicillin-binding protein (PBP) $2 \mathrm{~A}$ that confers resistance to all $\beta$-lactam antimicrobial agents. Such strains are designated methicillin-resistant $\mathrm{S}$. aureus (MRSA). PBP $2 \mathrm{~A}$ is encoded by the mecA gene cassette, that can be detected by PCR and other DNA-based methods. Recently, a novel mecA homologue mecCLGA251 has been described ${ }^{3}$. The novel mecA gene also confers resistance to $\beta$-lactam antibiotics but escapes detection by molecular diagnostic tests currently used for identification of $\mathrm{MRSA}^{3}$. Genetic techniques have been used to study the evolution of the MRSA clones that have emerged since the early 1960s, and to study their worldwide dissemination. The early MRSA clones were hospital-associated (HA-MRSA). However, there 
is now an evolution of community-associated MRSA (CA-MRSA) clones. CA-MRSA mostly causes skin or soft-tissue infections as well as deep-seated infections such as necrotizing pneumonia.

The culture characteristics of S. aureus on various selective media have served as the basis for its presumptive identification ${ }^{4}$. In an attempt to achieve presumptive isolation of pathogenic staphylococci from clinical samples in a single step, Mannitol Salt Agar (MSA) was developed in $1945^{5,6}$. Several modifications of MSA were subsequently described ${ }^{7,8}$. The key to the presumptive identification of $\mathrm{S}$. aureus on MSA is the selective growth in the presence of $5-6.5 \% \mathrm{NaCl}$ and yellow pigmented colonies resulting from fermentation of mannitol ${ }^{9}$.

The molecular identification of $\mathrm{S}$. aureus strains can be established by detecting the presence of staphylococci protein A, (spa). The spa typing method is based on sequencing of the polymorphic $\mathrm{X}$ region of the protein A gene present in all strains of Staphylococcus aureus ${ }^{10}$ Also, a rapid and accurate method for identification of microorganisms is the matrix-assisted laser desorption ionization-time of flight (MALDI-TOF) mass spectrometry. ${ }^{11}$

In some developing countries, S. aureus is usually identified by growth and production of yellow colonies on MSA and the presumptive identification may be confirmed by a rapid agglutination kit or tube coagulase test. However, it has been observed that some of the results are often misleading because organisms that were identified as S. aureus with these methods may not be S. aureus but other staphylococci. There is therefore the need to compare commonly used methods of identification in Nigeria with other identification methods to determine the degree of accuracy of these commonly used methods. The aims of this study therefore are to compare the diagnostic accuracy of growth on MSA, tube coagulase, latex agglutination slide test with presence of spa gene and MALDI TOF for identification of $\mathrm{S}$. aureus and to determine the resistance pattern of isolated S. aureus strains to selected antibiotics with screening for MRSA in the isolated S. aureus strains and determination of its susceptibility pattern.

\section{Methodology \\ Bacterial strains}

One hundred and eighty five non duplicated strains which were previously isolated from the nares of 185 healthy college students volunteers aged between 16 and 35 years of Niger Delta University, Ammasoma, Bayelsa State, (a state in South-South Nigeria) between January and August, 2011 had been presumptively identified as $\mathrm{S}$. aureus through growth and production of yellow colonies on MSA. MSA is regarded as a presumptive media in the identification of S. aureus and differentiation of coagulase-positive staphylococci from coagulase-negative staphylococci. For the present study, all the strains were repeatedly streaked on MSA (Oxoid, UK) and incubated for 48 hours at $37^{\circ} \mathrm{C}$. All strains that grew on MSA with characteristic yellow colonies were used in further tests.

\section{Identification of strains \\ Coagulase tests}

The strains that produced bound coagulase (BC) were detected in all strains that grew on MSA with yellow colonies by a rapid agglutination kit for identification of $\mathrm{S}$. aureus: (Slidex® Staph PLUS. bioMérieux, France) according to the manufacturer's instructions. Slidex Staph Plus is based on a triple detection system: blue latex particles sensitized with human fibrogen and monoclonal antibodies simultaneously detect clumping factor, Protein A and group-specific antigen bound to the S. aureus-specific peripheral structure. All strains that grew on MSA with yellow colonies were also inoculated into a tube containing horse plasma (SSI, Denmark) and incubated at $37^{\circ} \mathrm{C}$ for 4 hours. The tube coagulase test detects free cogulase produced by the staphylococci by coagulating plasma.

\section{Detection of Spa gene.}

The polymorphic X region of the staphylococcal protein A gene present in almost all S. aureus described by Harmsen et al, ${ }^{10}$ was amplified for all isolated strains in a total volume of $50 \mu \mathrm{l}$ by adding $1 \mu \mathrm{l}$ of a 1:500 dilution of genomic DNA, $1 \mu \mathrm{l}$ of $25 \mathrm{Mm} \mathrm{MgCl} 2,1 \mu \mathrm{l}$ of forward primer, $1 \mu l$ of reverse primer, $21 \mu \mathrm{l}$ of water and $25 \mu \mathrm{l}$ of AmpliTaq Gold ${ }^{\circledR} 360$ Mastermix into a 0.2$\mathrm{ml}$ PCR tube. Primers used were spa-1113F (5'-TAAAGACGATCCTTCGGTGAGC-3') and spa-1415R (5'-CAGCAGTAGTGCCGTTTGCT'T-3'). A negative control (plain mastermix) and a positive control (from our laboratory's S. aureus collection) were included. Tubes were capped and placed in a Veriti Thermal Cycler (Applied Biosystems). Thermal cycling conditions included an initial $10 \mathrm{~min}$ at $95^{\circ} \mathrm{C}$ followed by 32 cycles 
of $30 \mathrm{~s}$ at $94^{\circ} \mathrm{C}, 30 \mathrm{~s}$ at $55^{\circ} \mathrm{C}$, and $60 \mathrm{~s}$ at $72^{\circ} \mathrm{C}$; and a final extension at $72^{\circ} \mathrm{C}$ for $10 \mathrm{~min}$. PCR products were resolved by agarose $(1 \%)$ gel electrophoresis previously stained with GelRed (Biotium Inc, Hayward, CA, USA) and run at approximately $40 \mathrm{mAmp}$ for $45 \mathrm{~min}$. The molecular marker used was a pUC mix Marker 8. The size of the PCR products was 400-600 bp for the spa gene. This test is the first gold standard to compare the accuracy of tube and slide coagulase test and growth on MSA.

\section{MALDI mass spectrometry.}

Identification of all isolated strains was carried out by MALDI mass spectrometry. A thin smear of $18 \mathrm{~h}$ old culture was deposited on a MALDI plate. The samples were overlaid with $1 \mu \mathrm{l}$ of matrix solution (saturated solution of cyano-4-hydroxycinnamic acid in 50\% acetonitrile and $2.5 \%$ trifluoroacetic acid). The matrix sample was crystallized by air drying at room temperature. Measurements were performed with a Microflex mass spectrometer (Bruker Daltonik, Bremen, Germany) using FlexControl software (version 3.0). Spectra were recorded in the positive linear mode (laser frequency, $20 \mathrm{~Hz}$; ion source 1voltage, $20 \mathrm{kV}$; ion source 2 voltage, $18.4 \mathrm{kV}$; lens voltage, $9.1 \mathrm{kV}$; mass range, 2,000 to $20,000 \mathrm{Da}$ ). For each spectrum 240 shots in 40 -shot steps from different positions of the target spot were collected and analyzed.

Results of the pattern-matching process were expressed as proposed by the manufacturer with scores ranging from 0 to 3. For each isolate, the highest score of a match against a spectrum in the database was used for identification. Scores below 1.7 were considered not to have generated a reliable identification; a score of 1.7 was considered identification to genus, and a score of 2.0 was used for species identification. This test is the second gold standard to compare the accuracy of tube and slide coagulase test and growth on MSA

\section{Antibiotic susceptibility testing}

Antibiotic susceptibility testing of all S. aureus strains was performed by Kirby Bauer technique according to the European Committee on Antimicrobial Susceptibility Testing (EUCAST) guidelines (www.eucast.org) using standard antibiotic discs (Oxoid, UK namely: fusidic acid, linezolid, ampicillin, norfloxacin, rifampicin, penicillin, clindamycin, erythromycin, cefoxitin, tobramycin, trimethoprim and vancomycin were placed firmly on an agar plates by a disc dispenser. The plates were incubated at $37^{\circ} \mathrm{C}$ for $24 \mathrm{hrs}$ and examined for clear zones of inhibition around the discs. The diameter of inhibition was measured and compared with standard zones to determine resistance

\section{Detection of MRSA \\ Growth on MRSA plates}

All S. aureus strains that were resistant to cefoxitin were subcultured on a chromogenic medium, chromIDTM MRSA plate (bioMérieux, France). After incubation, strains that grew on the plates with green colouration were selected for further studies.

\section{SLIDEX MRSA detection}

All S. aureus strains that were resistant to cefoxitin were tested for the presence of Penicillin Binding Protein-2 (PBP-2). This was performed with Slidex MRSA Detection Kit (bioMérieux, France) according to the manufacturer's instructions. This is a slide agglutination assay that detects PBP2a in MRSA by use of latex sensitised with a monoclonal antibody directed against PBP2a

\section{Detection of MecA and coagulase gene}

PCR amplification of mecA/(mecC(LGA251)) gene. PCR assay was performed for all suspected MRSA strains to amplify a region of mecA including the novel homologue mecC (mecALGA251) according to García-Álvarez et al (3) . Primers were as follows: Fw, 5' TCACCAGGTTCAACY]CAAAA 3'; and Rv, 5' CCTGAATCW]GCTAATAATATTTC 3'.

PCR reaction contained $50 \mu \mathrm{l}$ reaction volume of AmpliTaq Gold® 360 Mastermix (2x stock including PCR buffer, TAQ-polymerase, $\mathrm{MgCl} 2$ and dNTPs) with 0.5 $\mu l$ of each primer (stock for $100 \mathrm{pmol} / \mu \mathrm{l}$ (LGA251) and $25 \mathrm{pmol} / \mu \mathrm{l}$ (Coa)) and $10 \mu \mathrm{l}$ template for each. The mixture was prepared as freezer-mix (ready-to-use just as add $10 \mu \mathrm{l}$ template to $40 \mu \mathrm{l} \mathrm{mix}$ freshly thawed).

A negative control, with no target DNA, was included in the PCR and run in the Veriti Thermal Cycler (Applied Biosystems) and a positive control the amplification programme consisted of an initial denaturation step at $95^{\circ} \mathrm{C}$ for $10 \mathrm{~min} ; 40$ cycles of denaturing at $95^{\circ} \mathrm{C}$ for $1 \mathrm{~min}$, annealing at $55^{\circ} \mathrm{C}$ for $1 \mathrm{~min}$ and extension at $72^{\circ} \mathrm{C}$ for $2 \mathrm{~min}$; and a final extension at $72^{\circ} \mathrm{C}$ for $5 \mathrm{~min}$. PCR products were resolved by agarose $(1 \%)$ gel electrophoresis previously stained with GelRed (Biotium Inc, Hayward, CA, USA) and run at approximately 40 mAmp for 45 min. The molecular marker used was a pUC mix Marker 8. The size of the PCR products were $356 \mathrm{bp}$ for the $\mathrm{mec} \mathrm{A} / \mathrm{mec} \mathrm{C}$ gene. 
Determination of Minimum Inhibitory Concentration (MIC)

The MIC of antibiotics were determined in the MRSA and selected methicillin susceptible S. aureus (MSSA) for comparison using Gram-positive (GP) AST P-580 antibiotics sensitivity cards (bioMérieux, France) by Vitek-2 apparatus (bioMérieux) according to the manufacturer's instructions. The system determined the susceptible and resistant strains according to the software program.

\section{Results}

The nasal carriage rate of $\mathrm{S}$. aureus strains in the studied bacterial population is 9\%. All 185 strains grew as yellow colonies on MSA and all the strains were identified by MALDI TOF as staphylococci. 168 were coagulase negative staphylococci (CNS) while 17 strains from the total strains were identified as $\mathrm{S}$. aureus.

The presence or absence of spa gene in the 185 strains were detected by PCR amplification of the gene. There was amplification only in 17 strains and the strains were identified as S. aureus by MALDI TOF (Table I). All the 185 strains were tested for ability to coagulate plasma using agglutination kit and tube coagulase test. All the strains identified by MALDI TOF as S. aureus were also tube-coagulase positive (Table I). Twenty four isolates were positive in agglutination kit test. Isolates positive in agglutination kit but negative in tube coagulase test were identified as S. xylosus. . Three strains had positive result in tube coagulase test, spa-PCR and identified as S. aureus by MALDI TOF but had negative result in slide agglutination test.

Table 1: Comparison of Accuracies of Identification Methods for Isolated $S$. aureus Strains

\begin{tabular}{cccc}
\hline Gold Standards & Other Tests & & \\
\hline MALDI and spa & Tube Coagulase & Slide agglutination & MSA \\
17 S. aureus & 17 S. aureus & 14 S. aureus & 185 P. S. aureus \\
168 CNS & 168 CNS & 171CNS & - \\
& Sensitivity 1 & 0.82 & 0.1 \\
& Specificity 1 & 0.95 & - \\
\hline
\end{tabular}

Note $=\mathrm{P}$ is presumptive identified S.aureus which is only correct for 17 out of 185 isolates

The studied S. aureus strains were generally susceptible to the tested antibiotics except penicillin and trimethoprim. All the tested strains were resistant to penicillin except 3 strains. $53 \%$ of the isolates were also completely resistant to trimethoprim with no zone of inhibition.
All the tested isolates were susceptible to clindamycin, vancomycin, fusidic acid, rifampicin and linezolid. $7 \%$ of the tested strains were resistant to erythromycin and cefoxitin while $24 \%$ of the tested strains were resistant to norfloxacin. (Table II).

Table II: Percentage Resistance of Isolated $S$. aureus strains to antibiotics

\begin{tabular}{lll}
\hline Antibiotics & $\begin{array}{l}\text { No of Resistant } \\
\text { Strains }\end{array}$ & $\begin{array}{l}\% \\
\text { Resistance }\end{array}$ \\
\hline Ampicillin & 3 & 18 \\
Norfloxacin & 4 & 24 \\
Penicillin & 13 & 77 \\
Erythromycin & 1 & 6 \\
Cefoxitin & 1 & 6 \\
Tobramycin & 2 & 12 \\
Trimethoprim & 9 & 53 \\
\hline
\end{tabular}


Only one MRSA strain was detected in the studied population and the MRSA carriage in the studied population is $0.5 \%$. The MRSA strain (S. aureus FA91) grew on chromIDTM MRSA plate, produced PBP-2 by Slidex MRSA Detection Kit and was confirmed as
MRSA by detection of mecA/C by PCR. Comparison of the susceptibility pattern of the isolated MRSA with 3 MSSA revealed that the isolated MRSA was relatively susceptible to tested antibiotics with resistance observed only to beta lactams and trimethoprim. (Table III).

\section{Table III: Comparison of Resistance Pattern of Isolated MRSA and Selected MSSA by Determination of MIC.}

\begin{tabular}{ccccc}
\hline & \multicolumn{4}{c}{ Minimum Inhibitory Concentration of MRSA and MSSA } \\
Antibiotics & MRSA FA91 & MSSA FA28 & MSSA FA213 & MSSA FA293 \\
\hline Cefoxitin screen & + & - & - & - \\
Penicillin & R $>0.5$ & R $>0.5$ & S & R $>0.5$ \\
Oxacillin & R 1 & S & S & S \\
TMP/SMX & R 160 & R $>320$ & S & R $>320$ \\
Gentamycin & S & R 16 & S & S \\
Tobramycin & S & R 16 & S & S \\
Levofloxacin & S & R 8 & S & S \\
Moxifloxacin & S & R 2 & S & S \\
Tetracycline & S & R 16 & S & S \\
\hline
\end{tabular}

Note- MRSA: Methicillin Resistant Staphylococcus aureus MSSA: Methicillin Susceptible Staphylococcus aureus

R- Resistant

S:-Susceptible

\section{Discussion}

Microbiologists in developing countries rely on biochemical tests and growth on selective media to identify pathogens. Often, these identifications are inaccurate resulting in inappropriate prescription and use of antibiotics for misidentified pathogens ${ }^{12}$. From the studied 185 organisms that grew with yellow colonies on MSA in this study, 168 are CNS while 17 were identified as S. aureus by MALDI TOF. Furthermore, the accuracy of identification of $\mathrm{S}$. aureus strains by coagulase tests was tested with amplification of spa gene and MALDI TOF. The spa gene is specific to $\mathrm{S}$. aureus and expression of this gene provides useful strain identification 10,13 . Identification using whole cell protein profiles by MALDI-TOF-MS has been found useful and accurate in identifying most bacterial strains including $\mathrm{S}$. aureus strains $^{11}$.

The tube coagulase test (horse plasma) is 100\% accurate in identifying S. aureus strains, however slide agglutination method is not only positive for $\mathrm{S}$. aureus but also for S. xylosus strains and three $\mathrm{S}$. aureus strains were negative in slide agglutination test. Therefore, the slide agglutination test and growth on MSA is inaccu- rate for sole identification of S. aureus while the tube coagulase test is totally accurate for identification of $\mathrm{S}$. aureus strains.

The S. aureus strains isolated from this study were generally susceptible to the tested antibiotics except trimethoprim. This observed susceptibility may be because the strains were isolated from healthy carriers in the community. The high rate of resistance to trimethroprim by S. aureus strains found in this study is very disturbing. The combination of trimethoprim and sulfamethozole (co-trimoxazole) is widely used for various infections in Nigeria because of its low cost compared with other antibiotics. It is also commonly used as prophylaxis for HIV infected patients who have a high occurrence in Nigeria. This may account for a possible higher selective pressure on the agent therefore, accounting for increased rate of resistance. Huovinen et al. ${ }^{14}$ observed a clear trend in the resistance to TMP-SMX with strains isolated in the developing world being more often resistant than the strains isolated in the developed countries. The clinical significance of this finding is that resistance to co-trimoxazole has been associated with concurrent resistance to other antibiotics resulting in multi-drug resistant pathogens. ${ }^{15}$. 
There are conflicting reports about the prevalence of MRSA strains in Nigeria. Ghebremedhin et al. ${ }^{1}$ reported a prevalence rate of $20.23 \%$ of MRSA and $47 \%$ of CA-MRSA in Nigeria, Taiwo et al. ${ }^{16}$ reported a prevalence rate of $29 \%$ while $1.4 \%$ prevalence rate was reported by Adesida et al. ${ }^{17}$. Only one MRSA was detected in this study. This shows a low prevalence rate of MRSA in the studied population.

The MRSA isolated in this study is resistant to trimethoprim and beta lactams while generally susceptible to other tested antibiotics. Interestingly, an MSSA strain was highly resistant to tested antibiotics while the studied MRSA was very susceptible to tested antibiotics. The MRSA has the susceptibility pattern of CA-MRSA according to Fridkin et al. ${ }^{18}$ who reported that CA-MRSA are generally susceptible to most antimicrobial agents with the exception of beta-lactams and erythromycins. Lack of multidrug resistance in an MRSA may be an indication that it is CA-MRSA.

This investigation confirms that coagulation of horse plasma in tube coagulase test is reliable and accurate identification method for identifying $\mathrm{S}$. aureus strains and that slide agglutination test is not totally accurate. It also confirms that growth with mannitol fermentation (yellow) colonies on MSA cannot differentiate between S. aureus and CNS colonies on MSA. This observation was confirmed by Adebayo et al., who observed growth of CNS on MSA. It also showed low prevalence of MRSA in the studied population. The high rate of resistance to trimethroprim by $\mathrm{S}$. aureus strains found in this study calls for control in the use of the antibiotics in Nigeria

\section{Conflict of interest disclosure}

There is no conflict of interest in the manuscript

\section{References}

1. Ghebremedhin B, Olugbosi MO, Raji AM, Layer F, Bakare RA, König B, König, W (2009) Emergence of a Community-Associated Methicillin-Resistant Staphylococcus aureus Strain with a Unique Resistance Profile in Southwest Nigeria. J Clin Microbiol 47(9): 2975-2980 2. von Eiff C, Becker K, Machka K, Stammer H, Peters G, for the Study Group (2001) Nasal carriage as a source of Staphylococcus aureus bacteremia. N Engl J Med 344: 11-16

3. García-Álvarez L, Holden MT, Lindsay H, Webb CR, Brown DF, Curran MD, Walpole E, Brooks K, Pickard DJ, Teale C, Parkhill J, Bentley SD, Edwards GF, Gir- van EK, Kearns AM, Pichon B, Hill RL, Larsen AR, Skov RL, Peacock SJ, Maskell DJ, Holmes MA (2011) Meticillin-resistant Staphylococcus aureus with a novel mecA homologue in human and bovine populations in the UK and Denmark: a descriptive study. Lancet Infect Dis 11(8): 595-603

4. Stoakes L, Reyes R, Daniel J, Lennox G, John MA, Lannigan R, Hussain Z (2006) Prospective comparison of a new chromogenic medium, MRSASelect, to CHROMagar MRSA and mannitol-salt medium supplemented with oxacillin or cefoxitin for detection of methicillinresistant Staphylococcus aureus. J Clin Microbiol 44: 637-639

5. Chapman GH (1945) The significance of sodium chloride in studies of staphylococci. J Bacteriol 50: 201203

6. Blair EB, Emerson JS, Tull AH (1967) A new medium, salt mannitol plasma agar, for the isolation of Staphylococcus aureus. Am J Clin Pathol 47:30- 39

7. Safdar N, Narans L, Gordon B, Maki DG (2003) Comparison of culture screening methods for detection of nasal carriage of methicillin-resistant Staphylococcus aureus: a prospective study comparing 32 methods. J Clin Microbiol 2003; 41:3163- PubMed ;3166

8. Smyth RW, Kahlmeter G (2005) Mannitol salt agarcefoxitin combination as a screening medium for methicillin-resistant Staphylococcus aureus. J Clin Microbiol 43:3797-3799

9. Adebayo Shittu, Johnson Lin, Donald Morrison and Deboye Kolawole (2006) Identification and molecular characterization of mannitol salt positive, coagulase-negative staphylococci from nasal samples of medical personnel and students. J Med Microb 55: 317-324 10. Harmsen D, Claus H, Witte W, Rothgänger J, Claus H, Turnwald D, Vogel U (2003) Typing of methicillin-resistant Staphylococcus aureus in a university hospital setting by using novel software for spa repeat determination and database management. J Clin Microbiol 41(12):5442-5448

11. Madhava Charyulu E., Arumugam Gnanamani and Mandal A.B. (2012) Identification and Discrimination of Methicillin Resistant Staphylococcus aureus Strains Isolated from Burn Wound Sites Using PCR and Authentication with MALDI-TOF-MS. J Microbiol 52(3) :337-345

12. Ayeni FA, Adeniyi BA, Ogunbanwo ST, Tabasco R, Paarup T, Peláez C, Requena T (2009) Inhibition of uropathogens by lactic acid bacteria isolated from dairy foods and cow's intestine in western Nigeria. Arch Microbiol 191 (8): 639-648 
13. Walker J, Borrow R, Edwards-Jones IV, Oppenheim BA, Fox AJ (1998) Epidemiological characterization of methicillin-resistant staphylococcus aureus isolated in the North West of England by protein A (spa) and coagulase (coa) gene polymorphisms. Epidemiol Infect 121: 507-514

14. Huovinen P, Sundstrom L, Swedberg, G, Skold O (1995) Trimethoprim and sulfonamide resistance. Antimicrob Agents Chermother 39: 279-289

15. Zhanel GG, Karlowsky JA, Harding GKM, Carrie A, Mazzulli T, Low DE. The Canadian Urinary Isolates Study Group, and Daryl J,Hoban DJ (2000) A Canadian National Surveillance Study of Urinary Tract Isolates from Outpatients; Comparison of the Activities of Tri-
methoprim-Sulfamethoxazole, Ampicillin, Mecillinam, Nitrofurantoin and Ciprofloxacin. Antimicrob. Agents Chermother. 44: 1089-1092

16. Taiwo SS, Bamidele M, Omonigbehin EA, Akinsinde KA, Smith SI, Onile BA, Olowe AO (2005) Molecular epidemiology of methicillinresistant Staphylococcus aureus in Ilorin, Nigeria. West Afr J Med 24:100-106 17. Adesida SA, Boelens H, Babajide B, Kehinde A, Snijders S, Van Leeuwen W, Coker A, Verbrugh H, van Belkum, A (2005) Major epidemic clones of Staphylococcus aureus in Nigeria. Microb Drug Resist 11:115-121 18. Fridkin SK, Hageman JC, Morrison M, Sanza LT, Como-Sabetti K, Jernigan JA (2005) Methicillin-resistant Staphylococcus aureus disease in three communities. N Engl J Med 352: 1436-1444 